\title{
Péptido natriurético cerebral tipo B como marcador de sobrecarga hemodinámica del conducto arterioso permeable en el recién nacido prematuro
}

\section{B-type brain natriuretic peptide as marker of hemodynamic overload of the patent ductus arteriosus in the preterm infant}

\author{
J. Rafael Parra-Bravo ${ }^{1 *}$, M. Teresa Valdovinos-Ponce ${ }^{2}$, Heladia García ${ }^{3}$, Juan C. Núñez-Enríquez, \\ M. Lourdes Jiménez-Cárdenas², Rodrigo Avilés-Monjaraz ${ }^{2}$ y Wendy Lavana-Hernández ${ }^{2}$
}

${ }^{1}$ Servicio de Cardiología Pediátrica; ${ }^{2}$ Servicio de Neonatología. Hospital General Tláhuac, Secretaría de Salud; ${ }^{3}$ Unidad de Investigación en Análisis y Síntesis de la Evidencia, Centro Médico Nacional (CMN) Siglo XXI, Instituto Mexicano del Seguro Social (IMSS); ${ }^{4}$ Unidad de Investigación en Epidemiología Clínica (UMAE), Hospital de Pediatría, CMN Siglo XXI Dr. Silvestre Frenk Freund, IMSS. Ciudad de México, México

\begin{abstract}
Resumen
Introducción: El ecocardiograma es el método de referencia para el diagnóstico del conducto arterioso permeable (CAP) hemodinámicamente significativo (CAP-hs) del recién nacido prematuro (RNP). El péptido natriurético tipo $B$ (BNP) puede ser útil en el diagnóstico y el manejo del CAP-hs. Objetivo: Evaluar la utilidad del BNP como marcador de sobrecarga hemodinámica del conducto arterioso permeable en el RNP con edad gestacional < 32 semanas o peso < 1500 gramos, e identificar el mejor punto de corte para los valores de BNP que mejor prediga un CAP con repercusión hemodinámica que requiera tratamiento farmacológico o quirúrgico. Método: Estudio retrospectivo, observacional y descriptivo de RNP < 32 semanas de gestación o peso < 1500 gramos en los que se realizó ecocardiograma y determinación del BNP. Análisis de muestra global y por subgrupos, en función del CAP-hs. Resultados: Se analizaron 29 pacientes. Se encontró una correlación significativa entre la relación CAP/peso y los valores del BNP (prueba de Spearman: 0.71; intervalo de confianza del 95\%: 0.45-0.87; $p<0.001$ ). El mejor punto de corte del BNP para predecir CAP-hs fue $486.5 \mathrm{pg} / \mathrm{ml}$, con una sensibilidad del $81 \%$ y una especificidad del $92 \%(p<0.001)$. Conclusión: El punto de corte del BNP identificado en el presente estudio se correlacionó con la presencia de CAP-hs.
\end{abstract}

Palabras clave: Conducto arterioso permeable. México. Péptido natriurético tipo B. Prematuridad.

\begin{abstract}
Introduction: The echocardiogram is the gold standard, in the diagnosis of the hemodynamically significant patent ductus arteriosus (hs-PDA) of the premature newborn (PNB). Type $B$ natriuretic peptide (BNP) may be useful in the diagnosis and management of CAP-hs. Objective: To assess the utility of BNP as a marker of hemodynamic overload of the patent ductus arteriosus in newborns with gestational age $<32$ weeks or weight $<1500$ grams, and to identify the best cut-off point for BNP
\end{abstract}

\section{Correspondencia:}

*José R. Parra-Bravo

E-mail: rpb.cardioped@gmail.com
Disponible en internet: 03-11-2020

Arch Cardiol Mex. 2021;91(1):17-24

www.archivoscardiologia.com CC BY-NC-ND (http://creativecommons.org/licenses/by-nc-nd/4.0/) 
levels that would best predict a PDA with hemodynamic impact requiring pharmacological and/or surgical treatment. Methods: Retrospective, observational and descriptive study of PNB $<32$ weeks gestation or weight $<1500$ grams, in which echocardiogram and BNP determination was performed. Analysis of the global sample and by subgroups, depending on the hs-PDA status was performed. Results: $A$ total of 29 patients were analyzed. A significant correlation was found between the PDA/weight ratio and BNP levels (Spearman: 0.71; 95\% confidence interval: 0.45-0.87; $p<0.001$ ). The best BNP cut-off point to predict CAP-hs was $486.5 \mathrm{pg} / \mathrm{ml}$ with a sensitivity of $81 \%$ and specificity of $92 \%(p<0.001)$. Conclusion: The BNP cut-off point identified in the present study was correlated with the presence of CAP-hs.

Key words: Patent ductus arteriosus. México. B-type natriuretic peptide. Prematurity.

\section{Introducción}

El conducto arterioso permeable (CAP) se ha asociado con una morbilidad significativa en los recién nacidos prematuros (RNP), y especialmente en los de muy bajo peso al nacer. En un CAP hemodinámicamente significativo (CAP-hs) existe un cortocircuito importante del circuito sistémico al pulmonar, que resulta en un aumento del flujo sanguíneo pulmonar y una reducción del flujo sanguíneo sistémico. El robo diastólico en la circulación sistémica puede ocasionar un alto riesgo de hemorragia intraventricular, enterocolitis necrosante y anormalidades en la perfusión renal ${ }^{1}$. El aumento del flujo sanguíneo pulmonar contribuye al empeoramiento del síndrome de dificultad respiratoria y más tarde a la displasia broncopulmonar.

La aparición de los síntomas depende de la magnitud del cortocircuito izquierda-derecha a través del CAP y de la capacidad del RNP para manejar la sobrecarga de volumen. Los hallazgos clínicos, como un soplo continuo y pulsos saltones, no siempre están presentes, en especial en los prematuros con muy bajo peso al nacer y soporte ventilatorio, donde el CAP podría ser "silencioso". A la fecha, las evaluaciones clínicas y radiológicas utilizadas para diagnosticar un CAP-hs tienen limitaciones sustanciales, y el tratamiento temprano del CAP-hs en los RNP ha demostrado que mejora la sintomatología, reduce la duración del soporte ventilatorio, disminuye la necesidad de ligadura quirúrgica y acorta los días de estancia hospitalaria ${ }^{2,3}$.

Actualmente, la ecocardiografía se considera el método de referencia para el diagnóstico de un CAP-hs y desempeña un papel esencial en la evaluación y el impacto de la circulación del RNP, así como en el diseño de opciones de tratamiento. Existe un claro consenso en cuanto a los criterios ecocardiográficos que deben ser evaluados 4 .

Los péptidos natriuréticos son sintetizados y liberados dentro de la circulación sanguínea por los cardiomiocitos, con propiedades natriuréticas y vasodilatadoras ${ }^{5}$. El péptido natriurético cerebral de tipo $B(B N P)$ es sintetizado en los ventrículos del corazón y liberado en respuesta a la sobrecarga de volumen y presión, por lo que desempeña un importante papel en la regulación del volumen de líquido extracelular ${ }^{6}$. Se ha estudiado como un posible marcador en el diagnóstico y el manejo de una hemodinámica significativa del CAP ${ }^{7}$. Se ha demostrado que la determinación de los valores de BNP en los primeros días de vida puede ser útil para evaluar el compromiso hemodinámico y contribuir en la toma de decisiones. Sin embargo, los valores de BNP sugeridos en estos estudios se encuentran dentro de un amplio rango, lo que dificulta la definición de un punto de corte absoluto ${ }^{8-10}$. Existe poca información sobre los valores normativos del BNP en los RNP sin CAP, lo que dificulta determinar si la prematuridad y su comorbilidad, como el síndrome de dificultad respiratoria, el miocardio inmaduro y otras afecciones, tienen efecto sobre las concentraciones séricas de BNP ${ }^{11,12}$. Los valores de BNP son más altos en los primeros 3 días de vida, con cifras mayores en los RNP que en los recién nacidos de término, pero disminuyen progresivamente y son similares hacia el mes de edad ${ }^{13-15}$.

El objetivo primario del presente estudio fue evaluar la utilidad del BNP como marcador de sobrecarga hemodinámica del CAP en el RNP con edad gestacional $<32$ semanas o peso < 1500 gramos. El objetivo secundario fue identificar el mejor punto de corte para las concentraciones séricas de BNP, que mejor prediga un CAP con repercusión hemodinámica que requiera tratamiento farmacológico o quirúrgico, o ambos.

\section{Método}

\section{Pacientes}

Estudio retrospectivo, observacional y descriptivo, realizado en la unidad de cuidados intensivos neonatales (UCIN) del Hospital General Tláhuac, Secretaría de Salud de la Ciudad de México, en el periodo comprendido entre julio de 2018 y junio de 2019. Se 
incluyeron 29 recién nacidos (16 mujeres y 13 hombres) con una edad gestacional $<32$ semanas y un peso al nacer $<1500$ gramos, dentro de las primeras 24 horas del nacimiento. Se excluyeron los recién nacidos con defectos cardiacos congénitos mayores, anomalías cromosómicas, sepsis confirmada, insuficiencia renal, hipertensión pulmonar persistente y muerte dentro de los 3 días posteriores a su ingreso a la UCIN. El estudio fue aprobado por el comité de investigación institucional y se obtuvo el consentimiento informado de los padres.

\section{Ecocardiograma}

A todos los RNP incluidos en el estudio se les efectuó un ecocardiograma transtorácico con Doppler color bidimensional entre el tercer y el quinto días de vida, para evaluación del CAP y descartar una cardiopatía congénita. Se utilizó un ultrasonido Phillips 5500 (Phillips Medical Systems, NA, Bothell, WA) con un transductor de $8 \mathrm{MHz}$ que incorpora flujo color y Doppler de onda continua y pulsada. Si se observó un CAP, el punto de constricción máxima del chorro de flujo de color se midió en milímetros, para evaluar el tamaño del conducto. Un CAP significativo se demostró por ecocardiografía tomando en cuenta la presencia de flujo ductal, con cortocircuito de izquierda a derecha (gradiente transductal $<20 \mathrm{mmHg}$ ) y flujo retrógrado en la aorta descendente. Para estimar la magnitud del cortocircuito de izquierda a derecha a través del CAP, los diámetros de la aurícula izquierda y la raíz aórtica se midieron en la vista paraesternal de eje corto a nivel de la válvula aórtica y se calculó la relación Ai/Ao (aurícula izquierda/aorta). Se midieron las dimensiones del ventrículo izquierdo en sístole y en diástole, para calcular la función sistólica por fracción de acortamiento y la fracción de eyección. Se midió el flujo regurgitante tricuspídeo para calcular la presión arterial pulmonar sistólica y se utilizó una vista sagital subcostal para obtener imágenes de la arteria mesentérica superior. La evaluación del CAP se realizó tomando en cuenta las recomendaciones del Neonatologist Performed Echocardiography (NPE) y la American Society of Echocardiography ${ }^{4,16,17}$.

Se diagnosticó CAP-hs si el RNP cumplió los criterios ecocardiográficos y tenía al menos tres de los siguientes seis signos clínicos de disfunción cardiovascular: 1) soplo sistólico o continuo en el borde paraesternal izquierdo; 2) hiperactividad precordial; 3) pulsos periféricos saltones; 4) taquicardia en reposo; 5) deterioro inexplicable del estado respiratorio; $y$
6) aumento del flujo pulmonar y cardiomegalia o signos de edema pulmonar en la radiografía de tórax. Los criterios ecocardiográficos fueron ${ }^{4,16:}$ 1) diámetro del CAP > $1.5 \mathrm{~mm}$; 2) relación Ai/Ao > 1.4; 3) turbulencia diastólica (cortocircuito de izquierda a derecha) en el Doppler de la arteria pulmonar; 4) relación diámetro del CAP/peso > $1.4 \mathrm{~mm} / \mathrm{kg}$; 5) flujo retrógrado $>30 \%$ de la diástole en la aorta descendente; y 6) velocidad del Doppler transductal $<2 \mathrm{~m} / \mathrm{s}$. Las mediciones las realizó un único observador, cardiólogo pediatra ecocardiografista con 20 años de experiencia en esta técnica. La concordancia intraobservador evaluada mediante el índice Kappa en el presente estudio fue del $100 \%$ en una submuestra de 15 estudios. Los prematuros con CAP-hs fueron tratados con paracetamol, ibuprofeno o cirugía, dependiendo de su estado clínico (grupo con intervención). Se realizó un segundo ecocardiograma transcurridas 72 horas para determinar si hubo cambios asociados al tratamiento.

\section{Medición de la concentración plasmática del BNP}

Se tomaron muestras de sangre $(0.5 \mathrm{ml})$ para BNP en plasma mediante aspiración con catéter arterial o venoso, o mediante muestreo de sangre venosa junto con otras muestras de sangre habituales para evitar punciones adicionales y un muestreo de sangre excesivo. La sangre se recogió coincidiendo en el tiempo con el ecocardiograma transtorácico. Las muestras se analizaron con el kit diagnóstico BNP $\left(\right.$ Alere $^{\mathrm{TM}}$ Triage $^{\circledR}$ BNP assay; Werfen Co, IL, USA) y los resultados se expresaron en $\mathrm{pg} / \mathrm{ml}$.

En los pacientes con sospecha de CAP se realizó interconsulta al servicio de cardiología pediátrica para corroborar el diagnóstico y el operador del estudio ecocardiográfico reportó los resultados, emitiendo recomendaciones terapéuticas. Los datos que incluyeron cambios en el peso corporal, el estado cardiorrespiratorio y la ingesta y salida de líquidos se recabaron cada tercer día, y los datos sobre la duración de la estadía y la prevalencia de enterocolitis necrosante, displasia broncopulmonar, hemorragia intraventricular y mortalidad se recabaron de los expedientes clínicos y de la base de datos de la UCIN.

\section{Análisis estadístico}

El análisis descriptivo se realizó utilizando el paquete estadístico SPSS (Statistics for Windows, versión 20.0), con cálculos de medidas de tendencia 
Tabla 1. Datos demográficos y parámetros clínicos de los recién nacidos prematuros y comparación de variables entre los subgrupos

\begin{tabular}{|c|c|c|c|c|}
\hline Parámetro & Grupo total $(n=29)$ & Subgrupo CAP - hs $(n=16)$ & Subgrupo CAP - nhs (n =13) & p \\
\hline Edad gestacional (sem)* & $29.0(3.9)$ & $27.6(5.3)$ & $29.0(2.5)$ & 0.46 \\
\hline Peso al nacer $(\mathrm{g})^{*}$ & $1080(350)$ & $990(403)$ & $1210(313)$ & 0.27 \\
\hline Sexo femenino ${ }^{\dagger}$ & $17(58.6)$ & $9(56.3)$ & $8(61.5)$ & 0.77 \\
\hline Uso de esteroide antenatal ${ }^{\dagger}$ & $15(51.7)$ & $7(43.8)$ & $8(61.5)$ & 0.34 \\
\hline Operación cesárea ${ }^{\dagger}$ & $24(82.7)$ & $4(25)$ & $1(7.7)$ & 0.61 \\
\hline Apgar 5 minutos* & $8(1.0)$ & $8(1.0)$ & $9(1.0)$ & 0.002 \\
\hline PA sistólica $(\mathrm{mmHg})^{\ddagger}$ f & $54 \pm 9$ & $53 \pm 7$ & $55 \pm 9$ & 0.32 \\
\hline PA diastólica $(\mathrm{mmHg})^{\ddagger}$ & $33 \pm 8$ & $28 \pm 6$ & $35 \pm 8$ & 0.001 \\
\hline Robo diastólico en $\mathrm{AoD}<30 \%^{\dagger}$ & $18(62.1)$ & $14(87.5)$ & $4(30.8)$ & 0.002 \\
\hline Aporte de líquidos (ml/kg/día $)^{\ddagger}$ & $147 \pm 31$ & $159 \pm 36$ & $142 \pm 29$ & 0.07 \\
\hline Diámetro del CAP (mm) & $\begin{array}{l}2.1(1.58)^{*} \\
2.2 \pm 0.9^{\ddagger}\end{array}$ & $\begin{array}{c}2.8(0.95)^{*} \\
2.84 \pm 0.69^{\ddagger}\end{array}$ & $\begin{array}{c}1.3(0.68)^{*} \\
1.42 \pm 0.41^{\ddagger}\end{array}$ & 0.0001 \\
\hline Relación CAP/peso (kg) & $\begin{array}{c}1.6(1.93)^{*} \\
1.99 \pm 1.14^{\ddagger}\end{array}$ & $\begin{array}{l}2.81(1.97)^{*} \\
2.69 \pm 1.1^{\ddagger}\end{array}$ & $\begin{array}{l}1.02(0.51)^{*} \\
1.13 \pm 0.29^{\ddagger}\end{array}$ & 0.0001 \\
\hline Valor sérico de BNP (pg/ml) & $\begin{array}{l}473(661.5)^{*} \\
684 \pm 890.1^{\mathrm{f}}\end{array}$ & $\begin{array}{c}751(817)^{*} \\
1061.9 \pm 105.7^{\text {f }}\end{array}$ & $\begin{array}{l}121.0(284.2)^{*} \\
219.9 \pm 227.8^{\ddagger}\end{array}$ & 0.0001 \\
\hline
\end{tabular}

AoD: aorta descendente; BNP: péptido natriurético cerebral tipo B; CAP: conducto arterioso permeable; CAP-hs: CAP hemodinámicamente significativo; CAP-nhs: CAP no hemodinámicamente significativo; PA: presión arterial.

*Mediana (rango intercuartílico).

†Número (\%).

`Media ( \pm desviación estándar).

centrai (media, mediana) y dispersión (desviación estándar, rangos intercuartílicos [RIQ]) para las variables cuantitativas de acuerdo con el tipo de distribución, y frecuencias y porcentajes para las variables cualitativas. La distribución de los datos se evaluó mediante su visualización con relación al histograma, así como de acuerdo con los valores de asimetría, curtosis y la prueba de Shapiro-Wilk, considerando valores de $p<0.05$ como una distribución no paramétrica. Utilizamos la prueba exacta de Fisher para comparar variables categóricas, y la prueba $U$ de Mann-Whitney para comparar variables continuas. Para la comparación de los subgrupos se utilizó la prueba $U$ de Mann-Whitney. Se consideró un valor $p<0.05$ para establecer la significancia estadística. Se utilizo la prueba de Spearman $\left(r_{s}\right)$ para correlacionar los valores de BNP y los hallazgos ecocardiográficos. Finalmente, se identificó mediante curvas ROC (receiver operating characteristic) el mejor punto de corte de los valores de BNP asociados con CAP-hs. Se determinaron el área bajo la curva (AUC), la sensibilidad y la especificidad para el mejor punto del corte.

\section{Resuitadios}

Durante el periodo de estudio se obtuvo una muestra de 29 RNP, con unas medianas de edad gestacional de 29.0 semanas (RIQ: 3.9 ) y de peso al nacer de 1080 gramos (RIQ: 350). Los datos demográficos y las características clínicas de los pacientes se exponen en la tabla 1, y el algoritmo de actuación diagnóstico-terapéutica se muestra en la figura 1.

Los pacientes se dividieron en dos subgrupos: el primero correspondiente a 16 pacientes con CAP-hs (mediana de edad gestacional: 27.6 semanas; mediana de peso: $990 \mathrm{~g}$ ) y el segundo con 13 pacientes con CAP no hemodinámicamente significativo (CAP-nhs) (mediana de edad gestacional: 29.0 semanas; mediana de peso: $1210 \mathrm{~g}$ ). No hubo diferencias significativas entre los subgrupos en cuanto a los datos relativos a edad gestacional, peso al nacimiento, sexo, uso de esteroide antenatal, líquidos administrados, presión sistólica sistémica ni tipo de parto.

La mediana del valor del BNP para el grupo total ( $n=29$ ) fue de $473 \mathrm{pg} / \mathrm{ml}$ (RIQ: 661.5) y la concentración del BNP en el subgrupo con CAP-hs fue significativamente más alta que en el subgrupo con CAP-nhs 


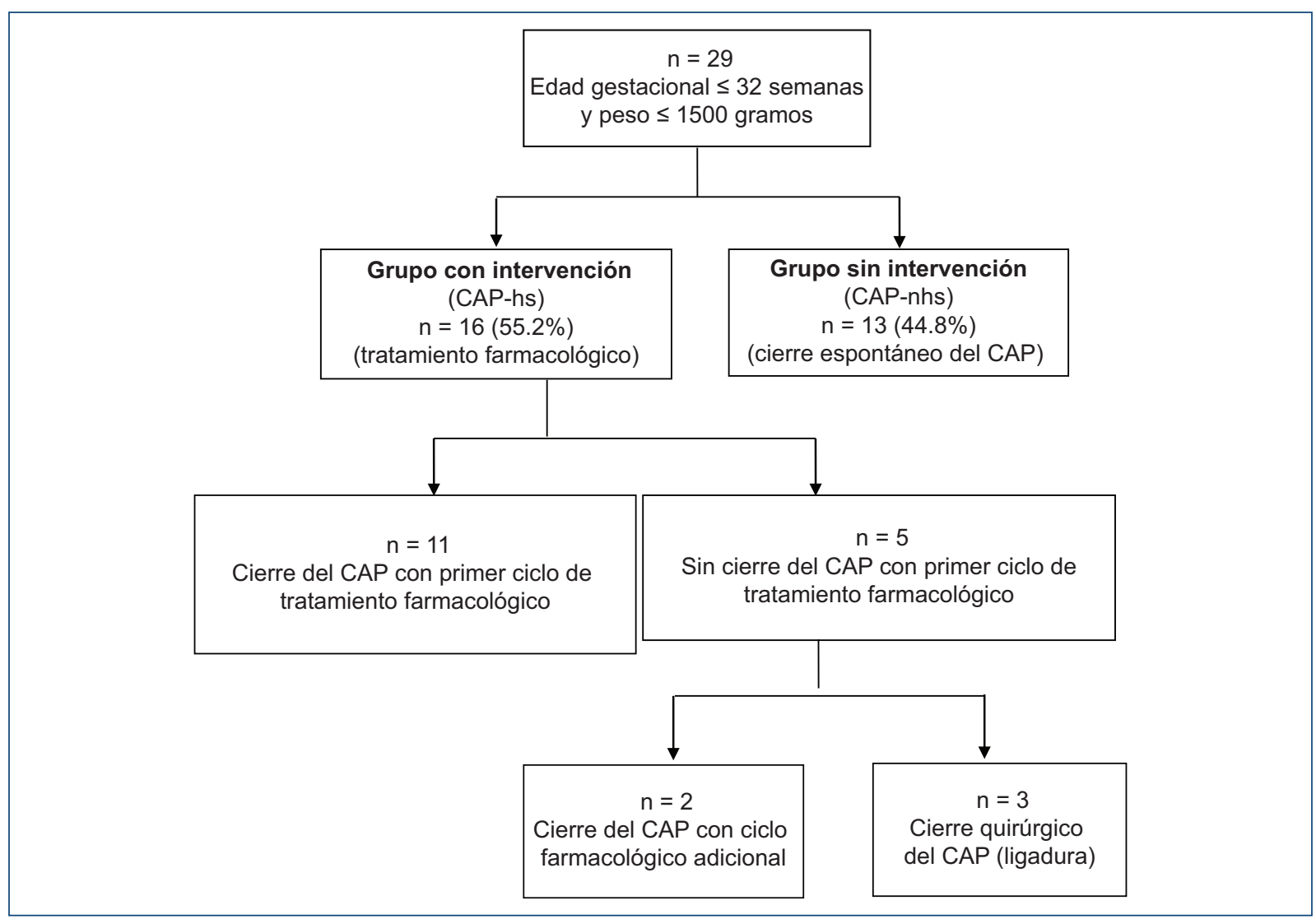

Figura 1. Diagrama de flujo de los pacientes incluidos en el estudio. CAP: conducto arterioso permeable; CAP-hs: CAP hemodinámicamente significativo; CAP-nhs: CAP no hemodinámicamente significativo.

(mediana de 751 vs. $121 \mathrm{pg} / \mathrm{ml} ; \mathrm{p}=0.0001$ ). Además, se observó significancia estadística en las variables de Apgar a los 5 minutos, presión arterial diastólica, robo diastólico en la aorta descendente, diámetro del CAP y relación CAP/peso en kilogramos. Como ya se ha mencionado, los casos con hipertensión pulmonar, sepsis o enfermedad cardiaca congénita fueron excluidos debido al posible incremento del valor del BNP no relacionado con CAP-hs.

El $71.7 \%$ de los pacientes mostró CAP en la primera evaluación ecocardiográfica, con una correlación significativa entre los hallazgos ecocardiográficos y el valor basal del BNP (Tabla 2). Se observó una correlación positiva entre la relación CAP/peso y valor del BNP $\left(r_{s}=0.71\right.$; intervalo de confianza del 95\% [IC95\%]: 0.45$0.87 ; p<0.001$ ) (Fig. 2). El mejor punto de corte del BNP para predecir CAP-hs fue de $486.5 \mathrm{pg} / \mathrm{ml}$, con una sensibilidad del $81 \%$, una especificidad del $92 \%$ y un AUC de 0.93 (IC95\%: 0.84-1.0; $p<0.001$ ) (Fig. 3).

Los pacientes del subgrupo con CAP-hs $(n=16)$ recibieron intervención (cierre farmacológico o cirugía): 10 con ibuprofeno (62.5\%), 6 con paracetamol (37.5\%) y finalmente 3 pacientes requirieron una ligadura quirúrgica debido a falla del tratamiento farmacológico. El cierre farmacológico del CAP se logró con un primer ciclo en 11 pacientes (68.7\%), mientras que en 2 pacientes el cierre se logró con un segundo ciclo (Fig. 1). En los pacientes con CAP-hs $(n=16)$ se observó una caída importante entre la mediana de los valores de BNP basales y finales (751.50; RIQ: 817 vs. 211.5; RIQ: $268 \mathrm{pg} / \mathrm{ml}$, respectivamente; $\mathrm{p}=0.01$ ), con un porcentaje de descenso del $69.5 \pm 18.3 \%$. En los pacientes que requirieron intervención quirúrgica, la mediana del valor inicial del BNP fue casi tres veces mayor (1800; RIQ: $2940 \mathrm{pg} / \mathrm{ml}$ ) en comparación con aquellos que solo requirieron tratamiento farmacológico (630; RIQ: $394.5 \mathrm{pg} / \mathrm{ml})$.

Los pacientes del subgrupo con CAP-nhs (sin intervención) mostraron una caída similar y progresiva en las medianas de los valores del BNP basales y finales (630; RIQ: 394.5 vs. 172.0; RIQ: 224 pg/ml, respectivamente; $p=0.002$ ), con un porcentaje de descenso del 
Tabla 2. Correlación entre los valores séricos de péptido natriurético cerebral tipo $\mathrm{B}$ y variables ecocardiográficas

\begin{tabular}{|l|c|c|}
\hline $\begin{array}{l}\text { Parámetros } \\
\text { ecocardiográficos }\end{array}$ & $\begin{array}{c}\text { Correlación con valores } \\
\text { iniciales de BNP (pg/mI) } \\
\mathbf{r}_{\mathbf{s}} \text { (IC95\%) }\end{array}$ & p \\
\hline Relación Ai/Ao & $0.64(0.38-0.81)$ & $<0.001$ \\
\hline Diámetro del CAP & $0.66(0.23-0.91)$ & 0.007 \\
\hline Diámetro AoD & $0.03(-0.47-0.56)$ & 0.91 \\
\hline Relación CAP/AoD & $0.84(0.49-0.96)$ & $<0.001$ \\
\hline Relación CAP/peso en kg & $0.71(0.45-0.87)$ & $<0.001$ \\
\hline Gradiente del CAP & $0.11(-0.56-0.73)$ & 0.69 \\
\hline $\begin{array}{l}\text { Relación flujo TSVI/flujo } \\
\text { AMS }\end{array}$ & $0.45(-0.08-0.80)$ & 0.09 \\
\hline Relación flujo VM/VT & $0.11(-0.40-0.68)$ & 0.69 \\
\hline $\begin{array}{l}\text { Robo diastólico en AoD } \\
>30 \%\end{array}$ & $0.35(-0.25-0.83)$ & 0.20 \\
\hline PSP $\times$ IIT & & $0.61(0.06-0.87)$ \\
\hline Aifo:al & 0.01 \\
\hline
\end{tabular}

Ai/Ao: aurícula izquierda/aorta; AMS: arteria mesentérica superior; $A 0 D$ : aorta descendente; BNP: péptido natriurético cerebral tipo B; CAP: conducto arterioso permeable; IC95\%: intervalo de confianza del 95\%; PSP × IIT: presión sistólica pulmonar por índice de insuficiencia tricuspídea; $r_{\text {s }}$ : prueba de Spearman; TSVI: tracto de salida del ventrículo izquierdo; VM: válvula mitral; VT: válvula tricúspide.

$69.5 \pm 19.1 \%$. Se corroboró el cierre espontáneo del CAP por ecocardiografía, con una media de edad de 2.1 semanas.

\section{Discusión}

A pesar de la alta prevalencia del CAP, en un $60-70 \%$ de los RNP, y en especial en los de muy bajo peso al nacer, no hay métodos universalmente aceptados para diagnosticar un CAP-hs, lo que aumenta el riesgo de desarrollar patologías propias de esta población y ser una causa importante de morbimortalidad ${ }^{3}$. Una de las principales razones de esta situación es la naturaleza dinámica del tejido ductal y su variada sensibilidad a sustancias vasoconstrictoras y vasodilatadoras en el RNP. Así mismo, los factores que alteran la resistencia vascular pulmonar y sistémica pueden influir en el grado de cortocircuito ductal, independientemente de su tamaño ${ }^{15,18}$. Las estrategias para conseguir su cierre son aún motivo de debate y continúa la controversia sobre los criterios de tratamiento y el momento idóneo de realizarlo ${ }^{19-21}$.

El RNP con CAP-hs puede no desarrollar signos clíni$\cos y$, si los desarrolla, suele ser a partir del tercer día de

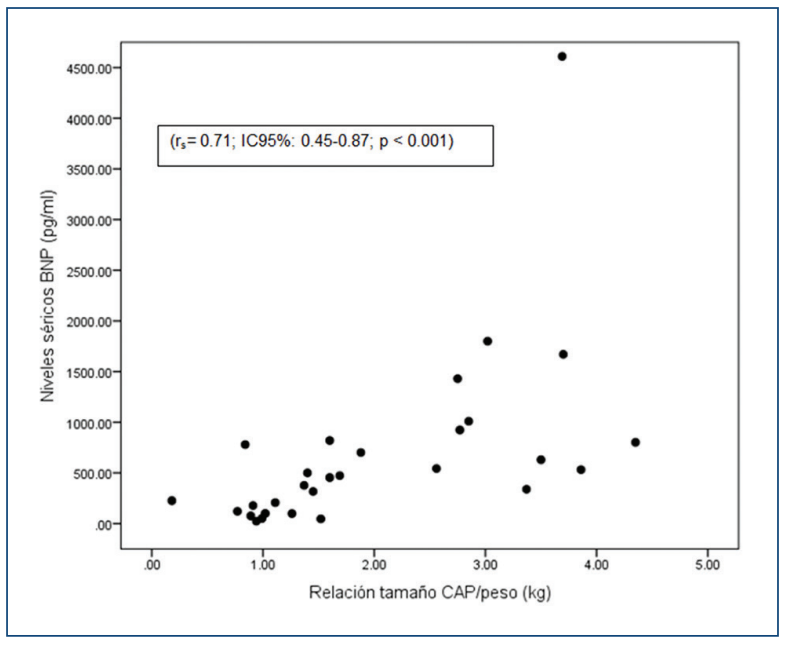

Figura 2. Correlación positiva entre la relación CAP/peso y el valor del péptido natriurético cerebral tipo $B$. BNP: péptido natriurético cerebral tipo $B$; CAP: conducto arterioso permeable; IC95\%: intervalo de confianza del $95 \% r_{s}$ : prueba de Spearman.

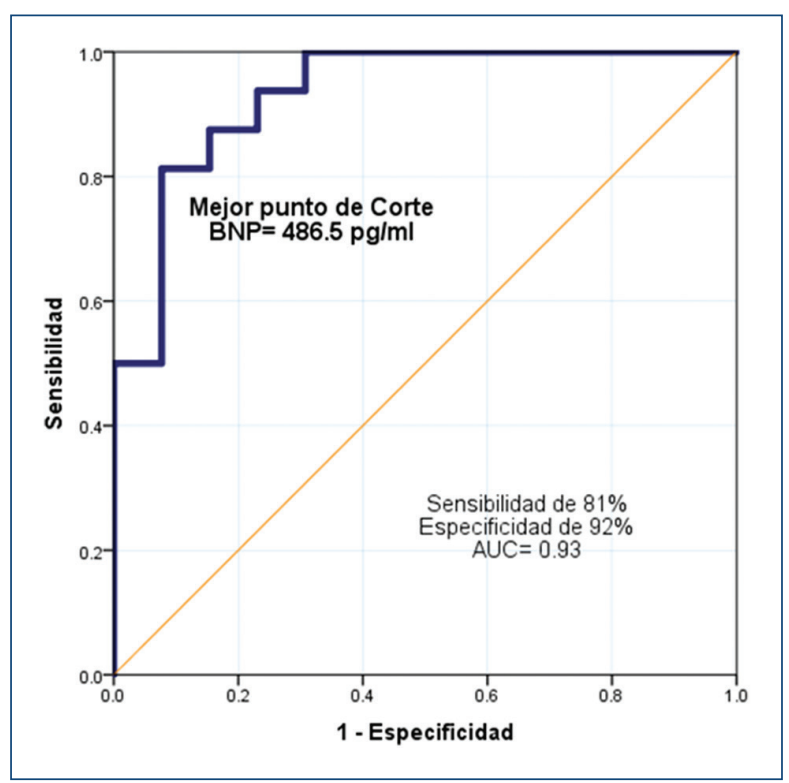

Figura 3. Curva ROC del valor del péptido natriurético cerebral tipo $B$ sérico en recién nacidos prematuros con conducto arterioso hemodinámicamente significativo (CAP-hs). Se calculó un punto de corte de $486.5 \mathrm{~g} / \mathrm{ml}$ para detectar un CAP-hs, basados en la curva ROC. BNP: péptido natriurético cerebral tipo B; AUC: área bajo la curva.

vida, cuando mejora la enfermedad pulmonar-3,18. Debido al retraso en la aparición de los síntomas clínicos, diversos autores han propuesto el tamizaje sistemático 
mediante ecocardiografía a las 48-72 horas de vida, tomando en cuenta que el estudio ecocardiográfico deberá incluir la evaluación de las características del CAP, los índices de sobrecirculación pulmonar en condiciones de carga del ventrículo izquierdo y los índices de hipoperfusión sistémica ${ }^{4}$. Hoy en día, la ecocardiografía es el principal método utilizado para detectar el CAP-hs en los $\mathrm{RNP}^{4,16,17}$. En el presente trabajo, el $71.7 \%$ de los pacientes presentaron CAP en el primer ecocardiograma, cifra superior a lo reportado22.

Algunos estudios han evaluado el uso potencial de los péptidos natriuréticos (marcadores cardiacos) en la medicina pediátrica y neonatal ${ }^{5}$. Aunque el BNP no reemplaza al ecocardiograma en el diagnóstico del CAP, puede obviar la necesidad de realizar ecocardiogramas repetidos para confirmar el cierre ductal postratamiento ${ }^{9,23}$. Los valores del BNP en los RNP son más altos que en los nacidos a término en los primeros días tras el nacimiento ${ }^{9-12}$. Su medición es un método que proporciona información inmediata y valiosa para el diagnóstico y el manejo del CAP en los RNP, porque sus valores son más altos en presencia de un CAP significativo ${ }^{13,15}$.

Diversos autores refieren que el BNP podría ser un parámetro fiable para predecir la ausencia o la presencia de $\mathrm{CAP}^{9-12}$; en nuestra muestra se encontró una fuerte asociación entre los valores del BNP y la presencia de CAP. En este trabajo, el $55.2 \%$ de los pacientes evidenció un CAP-hs con cifras superiores de BNP a las encontradas en aquellos con CAP-nhs dentro de los 3-5 días de vida ${ }^{23-25}$. Los RNP sin CAP o con CAP-nhs muestran variaciones considerables en la concentración sanguínea de BNP, con un pico máximo a las 24-48 horas de vida y una posterior caída hacía el quinto día de vida ${ }^{13-15}$. Cuando el CAP está presente en los RNP, la concentración de BNP es más alta y refleja la magnitud del cortocircuito a través del CAP, y puede considerarse que el BNP es capaz de diferenciar a aquellos con afectación hemodinámica ${ }^{10-12,25}$. No obstante, una de las limitaciones del presente trabajo fue que solo se evaluaron los valores del BNP y no los del NT-proBNP (propéptido natriurético cerebral amino-terminal), del cual se sabe que tiene unas mayores sensibilidad y especificidad (88 y $92 \%$ vs. 90 y $84 \%$, respectivamente $)^{8}$. Sin embargo, a la luz de los hallazgos del presente trabajo se puede evidenciar la posibilidad de que las asociaciones identificadas sean aún más fuertes si se evalúan mediante el marcador NT-proBNP en RNP24.

Se pudo elaborar una curva ROC para calcular el punto de corte del BNP y predecir un CAP-hs. El mejor valor de corte del BNP fue de $486.5 \mathrm{pg} / \mathrm{ml}$, con una sensibilidad del $81 \%$, una especificidad del $92 \%$ y un AUC de 0.93 (IC95\%: 0.84-1.0; $p<0.001$ ). Flynn, et al. ${ }^{9}$ mostraron que el mejor valor de corte del BNP fue de $300 \mathrm{pg} / \mathrm{ml}$. Una revisión sistemática para evaluar la seguridad diagnóstica del BNP en el CAP-hs menciona la amplia variedad de características de los pacientes y de los ensayos, con diversos valores de corte predictivos de diagnóstico y tratamiento ${ }^{8}$.

En consonancia con otras series ${ }^{6,22-25}$, observamos que el porcentaje de disminución de los valores de BNP se correlacionan bien con el cierre farmacológico del CAP, prediciéndolo con fiabilidad si esta caída es significativa. Así mismo, constatamos que cifras muy altas e iniciales de BNP se relacionan con una mayor incidencia de ligadura del conducto, aunque la utilidad clínica para predecir el tratamiento quirúrgico se ve limitada ${ }^{26-28}$.

\section{Conclusiones}

Las concentraciones séricas del BNP se relacionaron con la sobrecarga hemodinámica producida por el CAP en los RNP. Se pudo identificar un punto de corte óptimo del BNP con buena sensibilidad y especificidad para predecir CAP-hs.

La presencia de elevación del BNP apoyó los datos ecocardiográficos y la necesidad de tratamiento médico o quirúrgico, y su disminución informó de la buena sensibilidad que los valores de BNP muestran tras el cierre del CAP. Aunque la ecocardiografía seguirá siendo el método principal de diagnóstico, nuestros hallazgos permiten proponer al BNP como una herramienta adicional a esta en el diagnóstico y el seguimiento evolutivo del CAP.

\section{Financiamiento}

Los autores no recibieron ningún tipo de patrocinio para llevar a cabo este estudio.

\section{Conflicto de intereses}

Los autores declaran no tener ningún conflicto de intereses.

\section{Responsabilidades éticas}

Protección de personas y animales. Los autores declaran que para esta investigación no se han realizado experimentos en seres humanos ni en animales. 
Confidencialidad de los datos. Los autores declaran que han seguido los protocolos de su centro de trabajo sobre la publicación de datos de pacientes.

Derecho a la privacidad y consentimiento informado. Los autores declaran que en este artículo no aparecen datos de pacientes.

\section{Bibliografía}

1. Hamrick SE, Hansmann G. Patent ductus arteriosus of the preterm infant Pediatrics. 2010;125:1020-30.

2. Sasi A, Deorari A. Patent ductus arteriosus in preterm infants. Indian Pediatr. 2011;48:301-8.

3. Benitz WE; Committee on Fetus and Newborn, American Academy of Pediatrics. Patent ductus arteriosus in preterm infants. Pediatrics. 2016 137:10.1542/peds.2015-3730.

4. van Laere D, van Overmeire B, Gupta S, El-Khuffash A, Savoia M McNamara PJ, et al. Application of neonatologist performed echocardiography in the assessment of a patent ductus arteriosus. Pediatr Res. 2018;84:S46-S56.

5. Levin ER, Gardner DG, Samson WK. Natriuretic peptides. N Engl J Med. 1998:339:321-8

6. Chen S, Tacy T, Clyman R. How useful are B-type natriuretic peptide measurements for monitoring changes in patent ductus arteriosus shunt magnitude? J Perinatol. 2010;30:780-5.

7. Choi BM, Lee KH, Eun BL, Yoo KH, Hong YS, Son CS, et al. Utility of rapid $\mathrm{B}$-type natriuretic peptide assay for diagnosis of symptomatic paten ductus arteriosus in preterm infants. Pediatrics. 2005;115:e255-61.

8. Kulkarni M, Gokulakrishnan G, Price J, Fernandes C, Leeflang M, Pammi M. Diagnosing significant PDA using natriuretic peptides in preterm neonates: a systematic review. Pediatrics. 2015;135:e510-25.

9. Flynn PA da Graca RL, Auld PA, Nesin M, Kleinman CS. The use of bedside assay for plasma B-type natriuretic peptide as a biomarker in the management of patent ductus arteriosus in premature neonates. $J$ Pediatr. 2005;147:38-42.

10. Sanjeev S, Pettersen M, Lua J, Thomas R, Shankaran S, L'Ecuyer T. Role of plasma B-type natriuretic peptide in screening for hemodynamically significant patent ductus arteriosus in preterm neonates. J Perinatol. 2005;25:709-13.

11. Puddy VF, Amirmansour C, Williams AF, Singer DR. Plasma brain natriuretic peptide as a predictor of haemodynamically significant paten ductus arteriosus in preterm infants. Clin Sci (Lond). 2002;103:75-7.

12. Holmström H, Hall C, Thaulow E. Plasma levels of natriuretic peptides and hemodynamic assessment of patent ductus arteriosus in preterm infants. Acta Paediatr. 2001;90:184-91.
13. Mannarino S, Garofoli F, Mongini E Cerbo RM, Codazzi AC, Tzialla C, et al. BNP concentrations and cardiovascular adaptation in preterm and fullterm newborn infants. Early Hum Dev. 2010;86:295-8.

14. Koch $A$, Singer $H$. Normal values of $B$ type natriuretic peptide in infants, children and adolescents. Heart. 2003;89:875-8.

15. Tauber KA, Doyle R, Granina E, Munshi U. B-type natriuretic peptide levels normalise in preterm infants without a patent ductus arteriosus by the fifth postnatal day. Acta Paediatrica. 2016;105:e352-5.

16. Skelton R, Evans N, Smythe J. A blinded comparison of clinical and echocardiographic evaluation of the preterm infant for patent ductus arteriosus. J Paediatr Child Health. 1994;30:406-11.

17. Chiruvolu A, Punjwani P, Ramaciotti C. Clinical and echocardiographic diagnosis of patent ductus arteriosus in premature neonates. Early Hum Dev. 2009;85:147-9

18. Selimer A, Bjerre JV, Schmidt MR, McNamara PJ, Hjortdal VE, Host B, et al. Morbidity and mortality in preterm neonates with patent ductus arteriosus on day 3. Arch Dis Fetal Neonatal Ed. 2013;98:F505-10.

19. Noori S. Patent ductus arteriosus in the preterm infant: treat or not treat? J Perinatol. 2010;30(Suppl):531-7.

20. Urquhart DS, Nicholl RM. How good is clinical examination at detecting a significant patent ductus arteriosus in the preterm neonate? Arch Dis Child. 2003;88:85-6.

21. Sehgal A, McNamara PJ. Does echocardiography facilitate determination of hemodynamic significance attributable to the ductus arteriosus? Eur $\mathrm{J}$ Pediatr. 2009;168:907-14.

22. Acevedo-Olguín AL, Hernández-Benítez R, Iglesias-Leboreiro J Bernárdez-Zapata I, Gerardo-Del Hoyo MN, Rendón Macías ME. Functional echocardiography as a tool for the dynamic treatment evaluation in critically ill neonates. Arch Cardiol Mex. 2019;89:393-8.

23. Kim JS, Shim EJ. B-type natriuretic peptide assay for the diagnosis and prognosis of patent ductus arteriosus in preterm infants. Korean Circ J. 2012;42:192-6.

24. Montaner A, Galve Z, Fernández C, Jiménez L, Samper MP, Rite S. Utilidad del propéptido natriurético cerebral en el diagnóstico y manejo del ductus arterioso permeable. Ann Pediatr. 2017:86:321-8.

25. Mine K, Ohashi A, Tsuji S, Nakashima J, Hirabayashi M, Kaneko K. B-type natriuretic peptide for assessment of haemodynamically significant patent ducts arteriosus in premature infants. Acta Paediatrica. 2013; 102:e347-52.

26. Hsu JH, Yang SN, Chen HL, Tseng HI, Dai ZK, Wu JR. B-type natriuretic peptide predicts responses to indomethacin in premature neonates with patent ductus arteriosus. J Pediatr. 2010;157:79-84.

27. Lee JH, Shin JH, Park KH, Rhie YJ, Park MS, Choi BM. Can early B-type natriuretic peptide assays predict symptomatic patent ductus arteriosus in extremely low birth weight infants? Neonatology. 2013; 103:118-22.

28. Elsayed Y, Dakshinamurti S, Soni R, Ruffo I, Seshia MM. Does serum brain natriuretic peptide (BNP) together with a novel PDA score improve PDA treatment selection? Pediatr Res. 2011;70:643. 